\title{
Enhanced endoplasmic reticulum stress in bone marrow angiogenic progenitor cells in a mouse model of long-term experimental type 2 diabetes
}

\author{
Maulasri Bhatta ${ }^{1,2}$ - Jacey Hongjie Ma ${ }^{1,2,3}$ - Joshua J. Wang ${ }^{1,2}$. \\ Jonna Sakowski ${ }^{1,2} \cdot$ Sarah X. Zhang ${ }^{1,2,4,5}$
}

Received: 27 March 2015 / Accepted: 1 May 2015 /Published online: 11 June 2015

(C) Springer-Verlag Berlin Heidelberg 2015

\begin{abstract}
Aims/hypothesis Bone marrow-derived circulating angiogenic cells (CACs) play an important role in vascular repair. In diabetes, compromised functioning of the CACs contributes to the development of diabetic retinopathy; however, the underlying mechanisms are poorly understood. We examined whether endoplasmic reticulum (ER) stress, which has recently been linked to endothelial injury, is involved in diabetic angiogenic dysfunction.

Methods Flow cytometric analysis was used to quantify bone marrow-derived progenitors ( $\mathrm{Lin}^{-} / \mathrm{c}-\mathrm{Kit}^{+} / \mathrm{Sca}-1^{+} / \mathrm{CD} 34^{+}$) and blood-derived CACs $\left(\mathrm{Sca}-1^{+} / \mathrm{CD} 34^{+}\right)$in 15-month-old $\mathrm{Lepr}^{\mathrm{db}}$ $(d b / d b)$ mice and in their littermate control $(d b /+)$ mice used as a model of type 2 diabetes. Markers of ER stress in diabetic
\end{abstract}

M. Bhatta and J. Hongjie Ma contributed equally to this study.

Electronic supplementary material The online version of this article (doi:10.1007/s00125-015-3643-3) contains peer-reviewed but unedited supplementary material, which is available to authorised users.

Sarah X. Zhang

xzhang38@buffalo.edu

1 Department of Ophthalmology, University at Buffalo, State University of New York, 3435 Main Street, Buffalo, NY 14214, USA

2 SUNY Eye Institute, State University of New York, Buffalo, NY, USA

3 Aier School of Ophthalmology, Central South University, Changsha, China

4 Department of Biochemistry, University at Buffalo, State University of New York, Buffalo, NY, USA

5 Departments of Ophthalmology and Biochemistry, University at Buffalo, State University of New York, 3435 Main Street, Buffalo, NY 14214, USA $(d b / d b)$ and non-diabetic $(d b /+)$ bone marrow-derived early outgrowth cells (EOCs) and retinal vascular density were measured.

Results The numbers of bone-marrow progenitors and CACs were significantly reduced in $d b / d b$ mice. Vascular density was markedly decreased in the retinas of $d b / d b$ mice, and this was accompanied by vascular beading. Microglial activation was enhanced, as was the production of hypoxia inducible factor$1 \alpha(\mathrm{HIF}-1 \alpha)$ and vascular endothelial growth factor (VEGF). The production of ER stress markers (glucose-regulated protein-78 [GRP-78], phosphorylated inositol-requiring enzyme- $1 \alpha$ [p-IRE- $1 \alpha$ ], phosphorylated eukaryotic translation initiation factor- $2 \alpha$ [p-eIF $2 \alpha]$, activating transcription factor-4 [ATF4], C/EBP homologous protein [CHOP] and spliced Xbox binding protein-1 [XBP1s]) was significantly increased in bone marrow-derived EOCs from $d b / d b$ mice. In addition, mouse EOCs cultured in high-glucose conditions demonstrated higher levels of ER stress, reduced colony formation, impaired migration and increased apoptosis, all of which were largely prevented by the chemical chaperone 4-phenylbutyrate.

Conclusions/interpretation Taken together, our results indicate that diabetes increases ER stress in bone marrow angiogenic progenitor cells. Thus, targeting ER stress may offer a new approach to improving angiogenic progenitor cell function and promoting vascular repair in diabetes.

Keywords Diabetic retinopathy · Early outgrowth cells · Endoplasmic reticulum · Inflammation

$\begin{array}{ll}\text { Abbreviations } & \\ \text { ATF4 } & \text { Activating transcription factor-4 } \\ \text { ATF6 } & \text { Activating transcription factor-6 } \\ \text { BMMNC } & \text { Bone marrow mononuclear cell }\end{array}$




$\begin{array}{ll}\text { CAC } & \text { Circulating angiogenic cell } \\ \text { CHOP } & \text { C/EBP homologous protein } \\ \text { DR } & \text { Diabetic retinopathy } \\ \text { eIF2 } \alpha & \text { Eukaryotic translation initiation factor } 2 \alpha \\ \text { EOC } & \text { Early outgrowth cell } \\ \text { ER } & \text { Endoplasmic reticulum } \\ \text { FACS } & \text { Flow cytometry assisted cell sorting } \\ \text { FFA } & \text { Fundus fluorescein angiography } \\ \text { GCL } & \text { Ganglion cell layer } \\ \text { GRP78 } & \text { Glucose-regulated protein 78 } \\ \text { HG } & \text { High glucose } \\ \text { HIF-1 } \alpha & \text { Hypoxia inducible factor-1 } \alpha \\ \text { HSC } & \text { Haematopoietic stem cell } \\ \text { Iba-1 } & \text { Ionized calcium binding adaptor molecule-1 } \\ \text { IRE-1 } \alpha & \text { Inositol-requiring enzyme-1 } \alpha \\ \text { JNK } & \text { c-JUN N-terminal kinase } \\ \text { PBA } & \text { 4-Phenylbutyrate } \\ \text { PBMNC } & \text { Peripheral blood mononuclear cell } \\ \text { PERK } & \text { Protein kinase RNA-like endoplasmic } \\ & \text { reticulum kinase } \\ \text { qRT-PCR } & \text { Quantitative RT-PCR } \\ \text { Sca-1 } & \text { Stem cell antigen-1 } \\ \text { UPR } & \text { Unfolded protein response } \\ \text { VE-cadherin } & \text { Vascular endothelial-cadherin } \\ \text { VEGF } & \text { Vascular endothelial growth factor } \\ \text { VEGFR } & \text { Vascular endothelial growth factor receptor } \\ \text { XBP1 } & \text { X-box binding protein 1 } \\ \text { XBP1s } & \text { Spliced XBP1 } \\ \text { XBP1u } & \text { Unspliced XBP1 } \\ & \end{array}$

\section{Introduction}

Diabetic retinopathy (DR) is a common microvascular complication of diabetes that affects nearly all patients with type 1 diabetes and more than $60 \%$ of those with type 2 diabetes [1]. The rapidly increased prevalence of diabetes further renders DR a leading cause of blindness worldwide [2]. Retinal vascular degeneration is one of the early pathological hallmarks of DR, which eventually leads to retinal ischaemia and neovascularisation (NV) impeding vision. Deciphering the mechanisms of diabetes-associated vascular degeneration is critical for understanding the pathogenesis of DR.

Circulating angiogenic cells (CACs) are a specific population of progenitor cells derived from bone marrow haematopoietic stem cells (HSCs) and/or myeloid cells, or alternatively from the endothelium [3]. These cells exhibit a high propensity to navigate to sites of injury and integrate into or provide paracrine support to the damaged vasculature, thereby promoting vascular repair [4]. In diabetic patients, the numbers of CACs are reduced and their function is also impaired [5]. For example, CACs isolated from human patients with DR do not respond to hypoxia-regulated factors, such as stromal-derived factor 1 and vascular endothelial growth factor (VEGF) [6]. This, in turn, halts the recruitment of CACs into the injured blood vessels, a central event in vascular repair. Despite multiple contributing factors being identified in the pathogenesis of diabetes-induced CAC dysfunction $[7,8]$, we are still far from understanding the molecular pathways that regulate bone marrow angiogenic progenitor cells under normal and diabetic conditions.

The endoplasmic reticulum (ER) is the major organelle responsible for protein folding, lipid biosynthesis and $\mathrm{Ca}^{2+}$ storage. Recent studies have established that the ER also functions as an intracellular signalling platform to regulate cell fate and activity through the unfolded protein response (UPR) [9]. With ER stress, the UPR is activated by three ER transmembrane proteins: protein kinase RNA-like endoplasmic reticulum kinase (PERK), inositol-requiring enzyme-1 $\alpha$ (IRE- $1 \alpha)$ and activating transcription factor-6 (ATF6) (which have been reviewed elsewhere [10,11]). These UPR branches coordinate to regulate protein translation and transcription programmes to restore ER homeostasis [10, 11]. Unresolved ER stress activates proapoptotic and proinflammatory genes, such as those encoding C/EBP homologous protein (CHOP) and JNK, contributing to retinal inflammation and vascular dysfunction in ischaemic retinopathy and DR [12-14]. In endothelial cells, activation of the UPR plays a critical role in regulating cell proliferation, apoptosis and angiogenesis $[15,16]$. Interestingly, recent work by Li et al has implied ER stress as a key mediator of homocysteine-induced apoptosis in endothelial progenitor cells [17]. It is, however, still unknown whether ER stress contributes to diabetes-related abnormalities in CACs.

In the present study, we investigated how ER stress is involved in bone marrow-derived angiogenic progenitor dysfunction in experimental type 2 diabetes. We demonstrate, for the first time, that ER stress is increased in diabetic bone marrow-derived early outgrowth cells (EOCs) isolated from 15-month-old $d b / d b$ mice used as a model of type 2 diabetes. EOCs cultured under high glucose (HG) conditions also produce higher levels of ER stress markers, and an inhibition of ER stress significantly mitigates the HG-induced dysfunction and apoptosis of EOCs. These findings suggest that targeting ER stress may provide a novel approach to improving angiogenic progenitor cell function in diabetes.

\section{Methods}

Animals The study used 15-month-old $\operatorname{Lepr}^{d b}(d b / d b)$ mice and their littermate control $(d b /+)$ mice (Jackson Laboratories, Bar Harbor, ME, USA). All procedures were undertaken in strict agreement with the guidelines set out by Institutional Animal Care and Use Committees at the University at Buffalo, State University of New York, and with the Statement for the 
Use of Animals in Ophthalmic and Vision Research from the Association for Research in Vision and Ophthalmology.

Isolation and flow cytometry assisted cell sorting analysis of peripheral blood and bone marrow mononuclear cells Peripheral blood samples were collected via left ventricular puncture from deeply anaesthetised animals. Bone marrow was obtained by mincing femur and tibia in a sterile mortar in $5 \mathrm{mmol} / \mathrm{l}$ EDTA/PBS solution as previously described [18]. Peripheral blood mononuclear cells (PBMNCs) and bone marrow mononuclear cells (BMMNCs) were separated by density gradient centrifugation with Histopaque 1077 (MP Biomedicals, Solon, OH, USA) [18] and stained with the antibodies provided in the mouse haematopoietic and progenitor cell isolation kit (BD Biosciences, San Diego, CA, USA). This was followed by flow cytometry assisted cell sorting (FACS) analysis. For details, see electronic supplementary material [ESM] Methods.

\section{Immunohistochemistry, in vivo retinal imaging and fundal} fluorescein angiography For immunohistochemistry, mouse eyeballs were fixed in 4\% paraformaldehyde to prepare cryosections or retinal whole mounts for immunostaining (ESM Methods) using the antibodies described in ESM Table 1. Fundal photography and fundal fluorescein angiography (FFA) were performed on anaesthetised mice using a Micron III image system (Phoenix Research Laboratories, San Ramon, CA, USA). See ESM Methods for details.

Isolation, culture and characterisation of mouse bone marrow-derived EOCs Mouse EOCs were cultured as previously described [19]. Briefly, macrophage-depleted BMMNCs were seeded in fibronectin-coated plates and maintained in endothelial cell basal medium-2 supplemented with $5 \%$ FBS, VEGF-A, fibroblast growth factor, insulin-like growth factor-1, epidermal growth factor, ascorbic acid and antibiotics (Lonza, Walkersville, MD, USA). Non-adherent cells were removed after 4 days of culture and new medium was applied. EOCs, recognised as an attached cluster of spindle-shaped cells [20], were characterised on day 7 by immunostaining using primary antibodies against CD34, CD31, VEGFR2 and VE-cadherin (ESM Table 1). After incubation with Alexa Flour 488- or 594-conjugated secondary antibodies (Invitrogen, Carlsbad, CA, USA), the cells were visualised under an Olympus BX53 microscope (Olympus, Tokyo, Japan).

Analysis of colony-forming capacity of EOCs A total of $4 \times$ $10^{6} \mathrm{BMMNCs}$ from $d b /+$ and $d b / d b$ mice were seeded in each well of a six-well plate and cultured for 7 days. EOC colonies were identified as clusters of spindle-shaped cells. The number of colonies in nine random microscopic fields were counted per well. The results from three independent experiments were averaged and presented graphically. To investigate the effect of $\mathrm{HG}$ conditions and chemical chaperones on EOC colony formation, BMMNCs from 8-week-old C57/BL6 mice were cultured in $25 \mathrm{mmol} / 1$ glucose or mannitol, with or without $50 \mu \mathrm{mol} / 1$ sodium 4-phenylbutyrate (PBA) (Calbiochem, San Diego, CA, USA) for 7 days. The number of EOC colonies were quantified as described above.

Analyses of migration and apoptosis of EOCs The migratory function of EOCs was evaluated by a modified Boyden chamber (Transwell, Coster, Pittston, PA, USA) assay. Apoptosis of EOCs was determined using TUNEL assay with In Situ Cell Death Detection TMR red kit (Roche Diagnostic, Indianapolis, IN, USA) as previously described [21]. For details of the migration and TUNEL assays, see ESM Methods.

Western blot analysis Details are provided in ESM Methods.

Real-time qRT-PCR Total RNA was extracted using Trizol (Life Technologies, Carlsbad, CA, USA). Real-time qRT-PCR was performed using the iScript cDNA Synthesis Kit and SYBR ${ }^{\circledR}$ Green PCR Master Mix (Bio-Rad Laboratories, Hercules, CA, USA) according to the manufacturer's protocol. See ESM Methods for details.

Statistical analysis Data are expressed as mean \pm SD. Statistical analyses were performed using unpaired Student's $t$ test for two-group data and one-way ANOVA with Tukey's post hoc test for three groups or more. Statistical significance was accepted as $p<0.05$.

\section{Results}

Significant reduction in bone marrow progenitors and CACs in diabetic mice ESM Table 2 shows the blood glucose levels and body weights of the 15-month-old $d b / d b$ and $d b /+$ mice. BMMNCs were isolated and subjected to FACS analysis. Bone marrow progenitors were identified as Sca- $1^{+} /$ c-Kit ${ }^{+} / \mathrm{CD} 4^{+} / \mathrm{Lin}^{-}$cells (Fig. 1a-d) [22]. Compared with the non-diabetic controls, $d b / d b$ mice exhibited a $75 \%$ reduction in the number of bone marrow progenitors (Fig. 1e). In addition, the total number of BMMNCs was significantly reduced in $d b / d b$ mice compared with $d b /+$ mice (data not shown). These results, consistent with previous studies [23, 24], suggest an impairment of the bone marrow microenvironment in type 2 diabetes, resulting in decreased numbers of total BMMNCs and their subpopulations.

We next assessed CACs by subjecting PBMNCs to FACS analysis. CACs were identified as cells co-expressing Sca-1 and CD34 $\left(\mathrm{Sca}-1^{+} / \mathrm{CD} 34^{+}\right.$cells) [25-27]. In line with the 

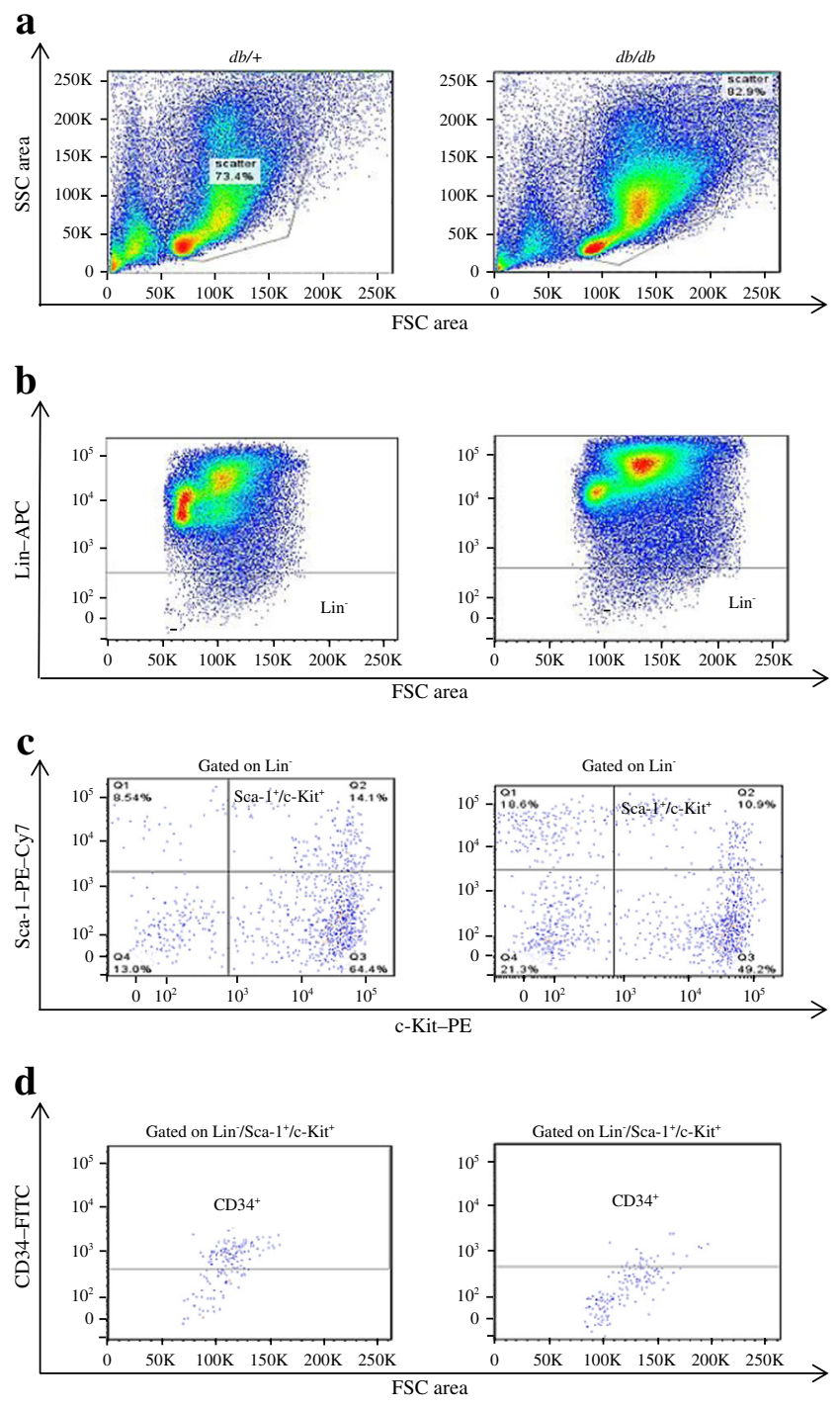

e
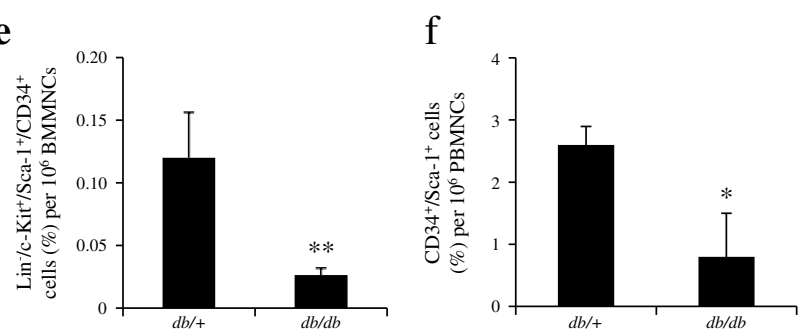

Fig. 1 Decreased numbers of bone marrow progenitors and CACs in $\mathrm{db}$ / $d b$ mice. (a-d) The gating strategy used to analyse the populations of BMMNCs by FACS analysis. (e) Frequencies of bone marrow progenitors $\left(\mathrm{Lin}^{-} / \mathrm{c}-\mathrm{Kit}^{+} / \mathrm{Sca}-1^{+} / \mathrm{CD} 34^{+}\right.$cells per $\left.10^{6} \mathrm{BMMNCs}[\%]\right)$. (f) Frequencies of CACs $\left(\mathrm{Sca}-1^{+} / \mathrm{CD} 34^{+}\right.$cells per $\left.10^{6} \mathrm{PBMNCs}[\%]\right)$. Data represent the mean $\pm \mathrm{SD}$ of three experimental replicates using six mice per group. ${ }^{*} p<0.05, * * p<0.01$, Student's $t$ test. APC, allophycocyanin; $\mathrm{Cy} 7$, cyanine 7; FSC, forward scatter; PE, phycoerythrin; SSC, side scatter

decreased number of bone marrow progenitors, $d b / d b$ mice demonstrated a $68 \%$ reduction in CAC number compared with age-matched $d b /+$ control animals (Fig. 1f).
Diabetic mice manifest significant retinal vascular degeneration The degeneration of retinal blood vessels is one of the salient features of DR [28]. We assessed changes in the retinal blood vessels using fundal photography and FFA with a Micron III imaging system. Compared with nondiabetic $d b /+$ mice (Fig. 2a-d), $d b / d b$ mice exhibited increased arterial reflection (Fig. 2e, red arrowheads), vascular beading (Fig. 2f) and a reduced retinal capillary density (Fig. 2g, h). To further confirm the degenerative changes in the retinal vasculature, retinal whole mounts were stained with isolectin B4 and the retinal vascular network was reconstructed from confocal series scans (Fig. 2i). Quantification of the results showed significant reductions in the retinal capillary density (Fig. 2j) and branch-points (Fig. 2k), indicating retinal vascular degeneration in $d b / d b$ mice.

Diabetic retinas are characterised by an inflammatory and ischaemic milieu Microglial activation has been associated with diabetic retinal changes in humans [29] as well as rodents [30]. Activated microglia produce proinflammatory cytokines (e.g. VEGF and TNF- $\alpha$ ) in early DR, promoting damage to the retinal neurons and vasculature [31]. To assess microglial activation, retinal flat mounts from $d b / d b$ mice were colabelled with isolectin B4 and Iba-1 [32] (ESM Fig. 1a). There was an $88 \%$ increase in the number of isolectin-positive microglia (ESM Fig. 1a, arrowheads) in the $d b / d b$ retinas (ESM Fig. 1b). Furthermore, in $d b / d b$ mice, an increased number of Iba-1-positive cells showed morphological changes characteristic of activated microglia, such as an enlarged soma and regional thickening and shortening of the processes, while the majority of microglia from the $d b+$ retinas displayed a resting phenotype with small perikarya and long, thin branched processes (ESM Fig. 1c).

VEGF is a growth factor released from ischaemic tissue, and hypoxia inducible factor- $1 \alpha$ (HIF- $1 \alpha$ ) is a critical transcription factor that induces VEGF during hypoxia and diabetes [33]. VEGF has been shown to recruit CACs to hypoxic tissues [34]. We examined the production of HIF- $1 \alpha$ and VEGF in the retinas of $d b / d b$ mice and, as expected, the levels of both HIF- $1 \alpha$ (ESM Fig. 1d) and VEGF (ESM Fig. 1e) were augmented in $d b / d b$ retinas. Increased immunoreactivity for HIF- $1 \alpha$ and VEGF was found predominantly in the inner retina of $d b / d b$ mice, although enhanced VEGF immunoreactivity was also seen in the outer segments of the photoreceptors (ESM Fig. 1e). Markedly increased HIF- $1 \alpha$ and VEGF production indicates the presence of tissue ischaemia in the inner retina of $d b$ / $d b$ mice.

We have previously shown that increased ER stress contributes to the upregulation of HIF-1 $\alpha$ and VEGF in diabetic retinas $[13,14]$. Here, we examined ER stress markers in the retina of $d b / d b$ and $d b /+$ mice. The results showed that the 
Fig. 2 Retinal vascular degeneration in $d b / d b$ mice. (a-h) Representative fundal images (a, e) showing increased arterial reflection in $d b / d b$ retinas (red arrowheads in $\mathbf{e}$ ) and FFA (b-d, f-h) showing vascular beading (the yellow frame in $\mathbf{f}$ ) and fewer capillaries (the red frame in $\mathbf{g}$, enlarged as h) in $d b / d b$ retinas. (i) Representative z-stack confocal images of the retinal vasculature in the GCL, inner plexiform layer (IPL) and outer plexiform layer (OPL) in retinal whole mounts stained with isolectin B4. Scale bars, $50 \mu \mathrm{m}$. (j, k) Quantification of capillary density (j) and branch-points (k) in the OPL layer. Data are shown as mean \pm $\mathrm{SD}$, four mice per group. ${ }^{* *} p<0.01, * * * p<0.001$, Student's $t$ test
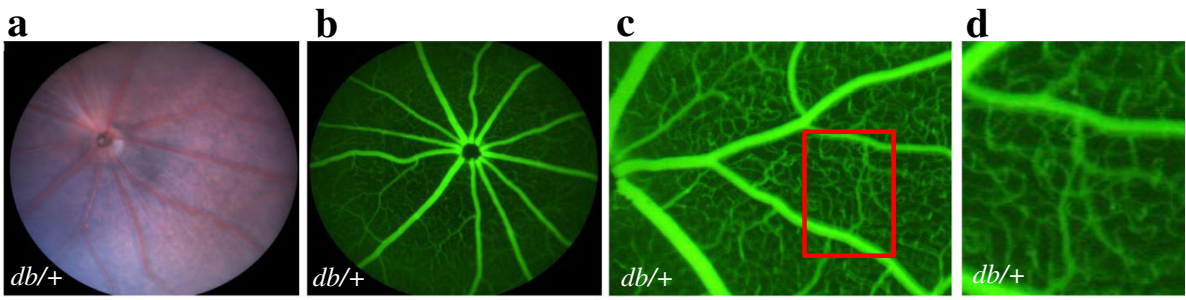

e
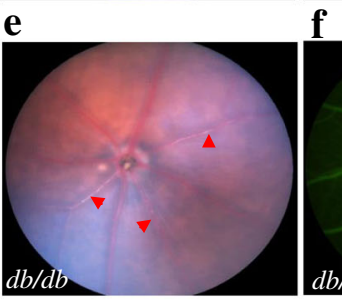

g

h
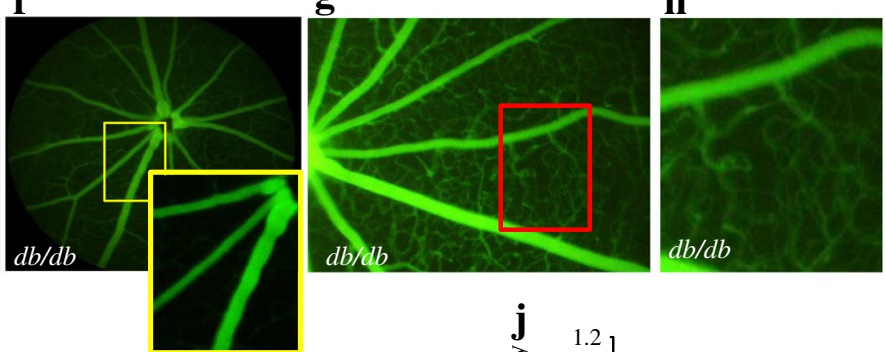

i
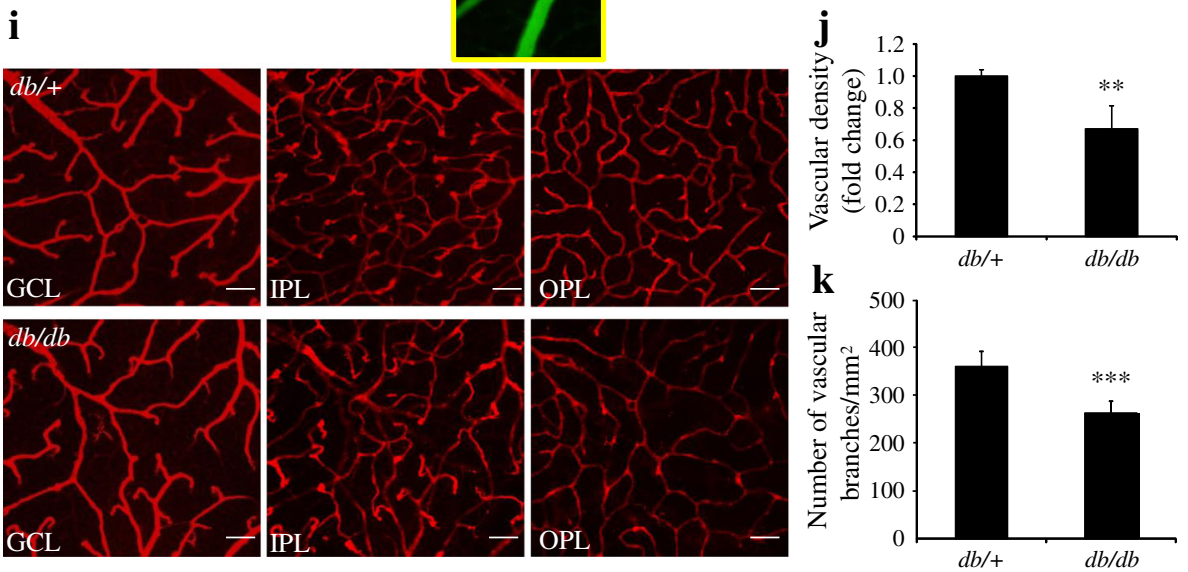

production of glucose-regulated protein-78 (GRP78) was increased in $d b / d b$ retinas (ESM Fig. 2a), especially in the ganglion cell layer (GCL) (ESM Fig. 2b) and inner segments of photoreceptors (ESM Fig. 2c). Consistent with this, the levels of p-IRE-1 $\alpha$ (ESM Fig. 2d and 2e), p-PERK (ESM Fig. 2f) and activating transcription factor-4 (ATF4; ESM Fig. 2g) were increased in $d b / d b$ retinas compared with $d b /+$ retinas. These results confirm that ER stress is increased in the retinas of $d b / d b$ mice.

Impaired colony-forming capability of diabetic bone marrow-derived EOCs To determine whether angiogenic progenitor cells are functionally abnormal in long-term diabetes, we assessed the colony-forming capacity of bone marrowderived EOCs from $d b / d b$ mice. We first characterised the EOCs by immunostaining using a stem cell marker (CD34) and endothelial cell markers (CD31, VEGFR2 and VEcadherin). At day 7, more than $90 \%$ of the cells showed dual staining with CD34 and CD31 (Fig. 3a). Moreover, VEGFR2 and VE-cadherin were also strongly expressed in EOCs (Fig. 3b, c), suggesting that these cells possessed the characteristics of angiogenic progenitor cells. The total numbers of colonies were quantified on day 7. As shown in Fig. 3d, e, $d b / d b$ EOCs displayed a significantly decreased colony- forming capacity compared to those from the $d b /+$ controls. These results suggest that the EOCs derived from long-term diabetic mice are dysfunctional.

Enhanced ER stress in diabetic bone marrow-derived EOCs ER stress has been reported to adversely affect the function of human CACs and predispose them to apoptosis [17]. Here, we examined ER stress markers by immunoblotting and real-time qRT-PCR in EOCs isolated from $d b / d b$ and control mice. As shown in Fig. 4a-g, the levels of ER chaperones (GRP78 and p58 $8^{\mathrm{IPK}}$ ), p-IRE- $1 \alpha$ and X-box binding protein-1 (XBP1) were significantly increased in diabetic EOCs. Moreover, a significant increase was observed in the production of p-eIF $2 \alpha$, ATF 4 and CHOP, suggesting a possible initiation of proapoptotic signalling. Consistent with the changes in protein levels, the gene expression of spliced $X b p 1$ $(X b p 1 s)$, total Xbpl and Chop was also elevated in diabetic EOCs (Fig. 4h-j). No significant difference was observed in the levels of ATF6 and p-JNK. Collectively, these results reveal, for the first time, enhanced ER stress in bone marrowderived EOCs in diabetes.

HG conditions induce ER stress and apoptosis in bone marrow-derived EOCs It has previously been reported that 
Fig. 3 Reduced colony formation in bone marrowderived EOCs from $d b / d b$ mice. $(\mathbf{a}-\mathbf{c})$ Characterisation of EOCs after 7 days of culture by immunostaining for CD31 and CD34 (a), VEGFR2 (b) or VE-cadherin (c). Scale bars, $50 \mu \mathrm{m}$. (d) Representative images of colony formation in bone marrow-derived EOCs from $d b / d b$ and $d b /+$ mice. Scale bars, $100 \mu \mathrm{m}$. (e) Total number of EOC colonies (mean $\pm \mathrm{SD}$, six mice per group in three experimental replicates). ${ }^{* * *} p<0.001$,

Student's $t$ test
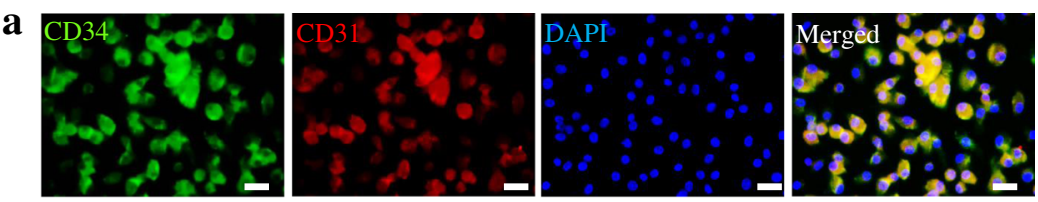

b
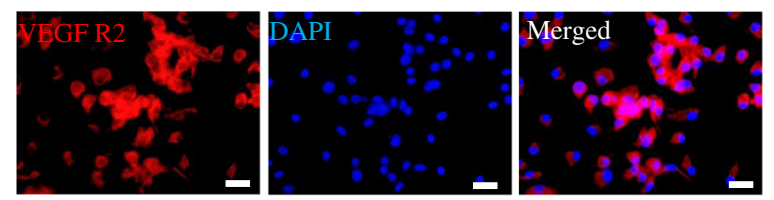

c
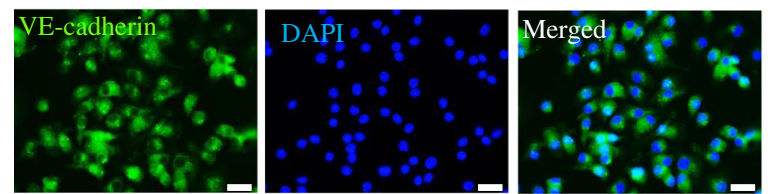

\section{d}

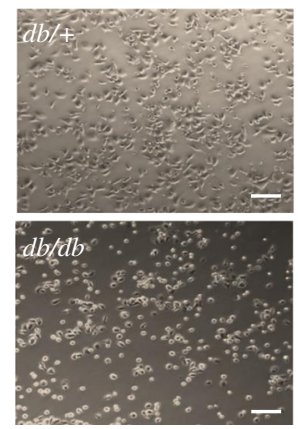

e

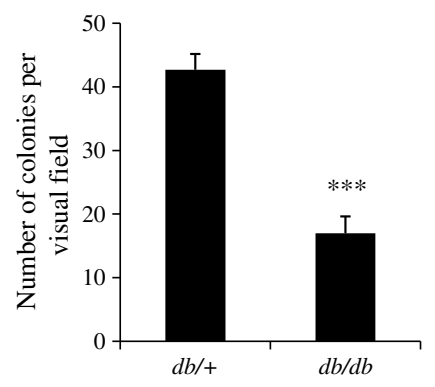

Fig. 4 Production of ER stress markers in bone marrow-derived EOCs. EOCs from $d b /+$ or $d b / d b$ mice were cultured for 12 days. (a-g) Protein levels of ER stress markers measured by western blot analysis and densitometry. (h-j) mRNA levels of Xbpls (h), total $X b p 1$ (i), and Chop (j). Results represent mean \pm SD of three independent experiments using six mice per group. ${ }^{*} p<0.05$, ${ }^{* *} p<0.01, * * * p<0.001$,

Student's $t$ test a
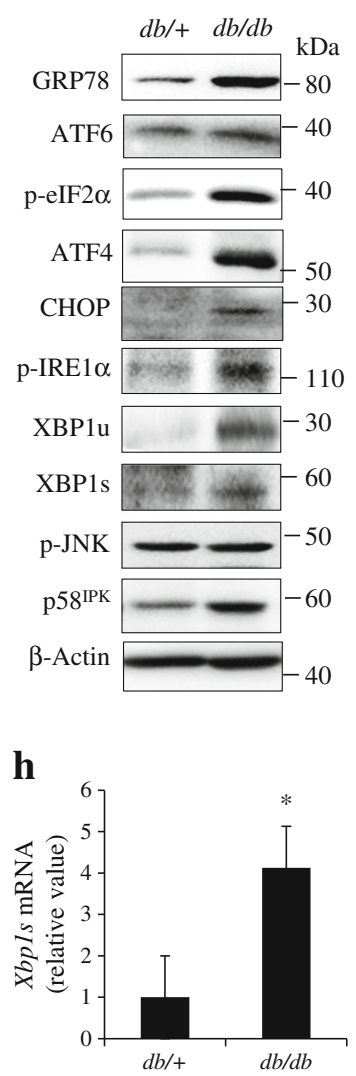

b
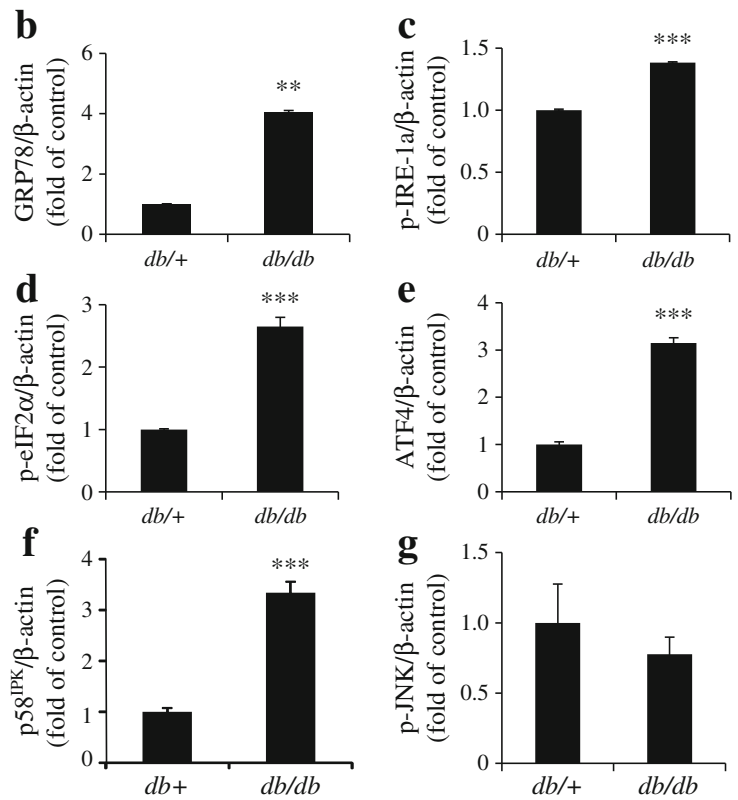

i

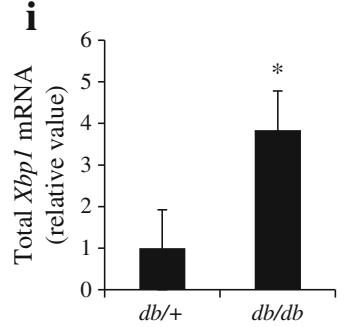

j

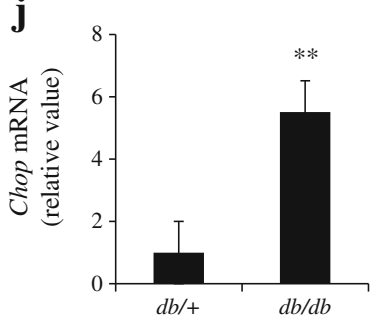


EOCs cultured under HG conditions display reduced colony formation, decreased proliferation and impaired migration [35]. To determine whether ER stress is involved in HGinduced EOC dysfunction, we examined ER stress markers in EOCs cultured in HG for 7 days. The apoptotic mediator cleaved caspase-3 was also measured to determine its correlation with apoptosis. Our results show that the protein levels of ATF6, spliced XBP1 (XBP1s), ATF4 and CHOP significantly increased in HG-treated, but not in mannitol-treated (osmotic control), EOCs (Fig. 5a, c-f), suggesting that HG enhances ER stress and activates all three branches of the UPR. Interestingly, HG-treated EOCs demonstrated a modest reduction in GRP78 (Fig. 5b). Similarly, higher levels of p-eIF2a, which regulates protein translation, were observed in diabetic but not HG-treated EOCs. Coincident with enhanced ER stress, the level of cleaved caspase-3 was significantly increased after HG treatment (Fig. 5g), indicating activation of caspase-3 and apoptosis.

Inhibition of ER stress alleviates HG-induced EOC dysfunction and apoptosis To further determine the role of ER stress in HG-induced dysfunction and apoptosis in EOCs, the EOCs were incubated with $\mathrm{HG}$ for 7 days in the presence or absence of PBA, a chemical chaperone widely used to facilitate protein folding and inhibit ER stress [14]. As expected, PBA treatment almost completely blunted the HG-induced increase of XBP1s, significantly reduced CHOP production and attenuated caspase- 3 activation, indicating a reduction of ER stress (Fig. 6a-e). Interestingly, PBA increased the level of p-eIF $2 \alpha$ in HG-treated cells, but had no effect on GRP78 production. In agreement with previous findings [35], HG treatment significantly reduced the colony-forming capacity of the EOCs, but this occurred to a much lesser extent in the PBA-treated cells (Fig. 6f, g). Moreover, HG-treated EOCs failed to migrate in response to the chemoattractant VEGF, and this was largely restored by PBA treatment (Fig. 6h, i). Furthermore, consistent with the reduction in caspase- 3 activation, PBA markedly alleviated the HG-induced apoptosis of EOCs (Fig. 6j, k). These results suggest that ER stress plays a critical role in HG-induced cell death and the dysfunction of bone marrow-derived angiogenic progenitors.

\section{Discussion}

Over the past decade, the role of bone marrow-derived progenitors and CACs in the maintenance of retinal blood vessels in physiological and diseased conditions has attracted significant attention $[18,36]$. Studies have shown that dysfunction and/or dysregulation of these cells contributes to diabetic microvascular complications [25, 36, 37]. A decreased frequency of CACs has been observed in 2-4-month-old type 1 and type 2 diabetic mice $[25,38]$ as well as in 6-12-month-old type 1 diabetic mice [24]. In the present study, we have reported that the number of bone marrow progenitors and CACs is reduced in 15-month-old $d b / d b$ mice. These changes correlate with enhanced vascular degeneration as well as greater levels of ER stress in bone marrow-derived EOCs in diabetic mice. Moreover, EOCs cultured under HG conditions exhibited increased ER stress, dysfunction and apoptosis, all of which were ameliorated by chemical chaperone treatment to reduce ER stress. These findings therefore collectively suggest an important role of ER stress in the pathogenesis of the reduction and functional disturbance of angiogenic progenitor cells that is seen in diabetes.

Mounting evidence demonstrates that diabetic CACs are dysfunctional in vascular repair [24, 25, 37]. Healthy human $\mathrm{CD}_{3} 4^{+}$cells promote the revascularisation of skin wounds [39] and accelerate the restoration of blood flow in type 1 diabetic mice [40]. In contrast, $\mathrm{CD} 34^{+}$cells from diabetic
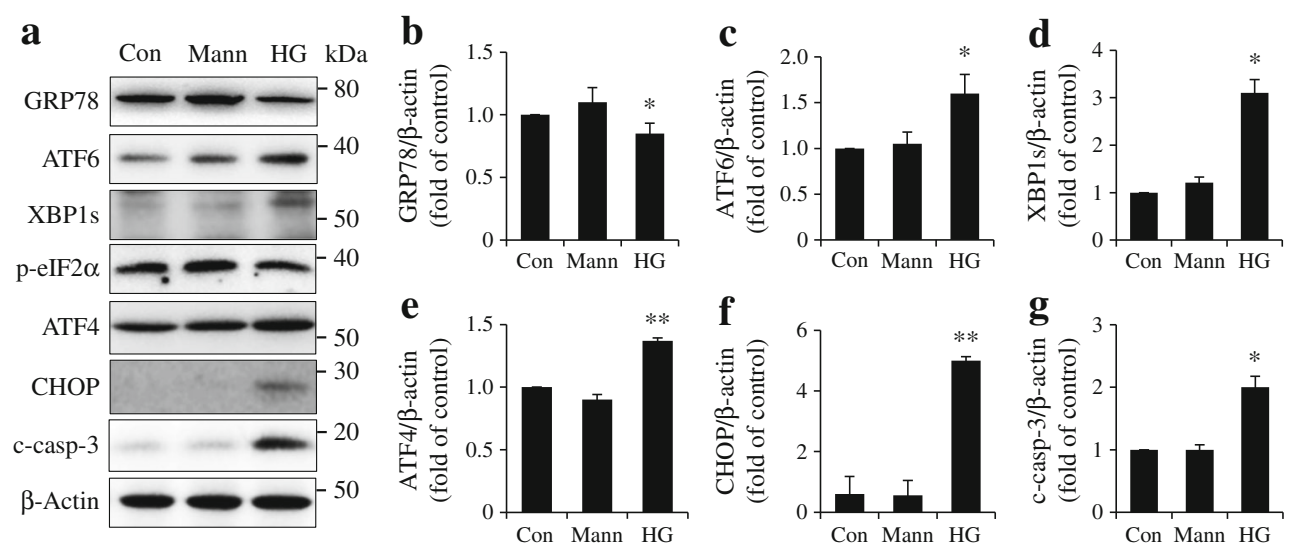

Fig. 5 Induction by $\mathrm{HG}$ conditions of ER stress and apoptosis in bone marrow-derived EOCs. BMMNCs from 8-week-old C57/BL6J mice were differentiated into EOCs by culturing for 7 days. For the entire duration of the culture, these were treated with $5 \mathrm{mmol} / \mathrm{l}$ glucose (Con), $25 \mathrm{mmol} / 1$ glucose $(\mathrm{HG})$ or $5 \mathrm{mmol} / 1$ glucose $+20 \mathrm{mmol} / \mathrm{l}$ mannitol

(Mann) for 7 days. Protein levels of ER stress markers and cleaved caspase-3 (c-casp-3) were determined by western blot analysis and densitometry. Results represent the mean $\pm \mathrm{SD}$ of three independent experiments. ${ }^{*} p<0.05,{ }^{* *} p<0.01$ vs mannitol, one-way ANOVA with Tukey's post hoc test 
Fig. 6 Inhibition of ER stress alleviates HG-induced dysfunctionality and apoptosis in EOCs. BMMNCs were differentiated into EOCs by culturing for 7 days. For the entire duration of the culture, these were treated with $5 \mathrm{mmol} / \mathrm{l}$ glucose (Con), $25 \mathrm{mmol} / 1$ glucose (HG) or $5 \mathrm{mmol} / \mathrm{l}$ glucose $+20 \mathrm{mmol} / \mathrm{l}$ mannitol (Mann) with or without $50 \mu \mathrm{mol} / \mathrm{l}$ of PBA for 7 days. (a-e) Protein levels of ER stress markers and cleaved caspase- 3 (c-casp-3) determined by western blot analysis. (f, g) Colony formation of EOCs after 5 days of culture. (h, i) Migration assay of EOCs, with the migrated cells stained with DAPI. (j, k) Apoptosis determined by TUNEL assay. Scale bars, $100 \mu \mathrm{m}$. Data represent mean $\pm \mathrm{SD}$ of three independent experiments. ${ }^{*} p<0.05,{ }^{* *} p<0.01$, $* * * p<0.001$, one-way ANOVA with Tukey's post hoc test
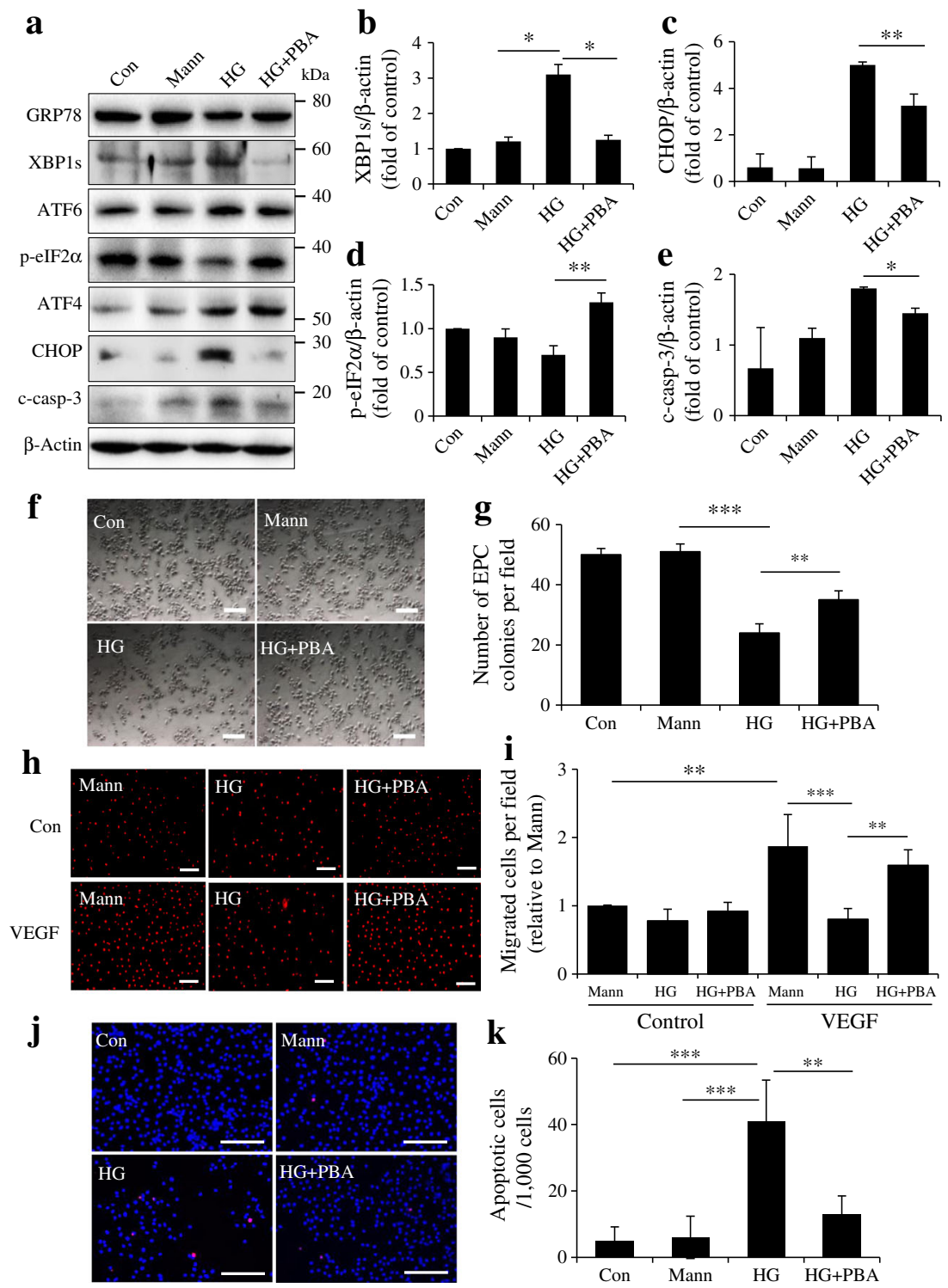

patients show impaired chemotactic and proangiogenic activity [37], defective adhesion, decreased proliferation and aberrant tubule formation [5]. Similarly, bone marrow-derived EOCs from 10-14-week-old $d b / d b$ mice are less effective in promoting wound closure in diabetic mice [25]. The results of our study confirm that the numbers of circulating CACs and bone marrow progenitors are significantly reduced in longterm experimental type 2 diabetes. The lack of CACs results in an insufficient source of angiogenic cells to repair any damaged blood vessels. This may partly explain the persistence of vascular degeneration in diabetic retinas, despite the presence of severe ischaemia and high levels of chemoattractant factors such as VEGF. Furthermore, in line with previous reports [35], our results show that bone marrow-derived EOCs cultured under HG conditions and EOCs isolated from $d b / d b$ mice exhibit significantly reduced colony-forming capacity. Moreover, HG-treated EOCs do not respond to VEGF in the migration assay. These results suggest that the functional aberrancy of EOCs also contributes to the failure of vascular repair during diabetes and the development of vascular degeneration.

Increased ER stress and activation of the UPR have been demonstrated in diabetic rodent retinas and are causally linked to inflammation and vascular leakage [12-15]. Enhanced ER stress has also been observed in retinal tissue from type 2 diabetic patients with non-proliferative retinopathy, suggesting a potential role of ER stress in the early development of DR [41]. In the present study, we observed increased ER stress coincident with significantly enhanced microglial activation and vascular degeneration in the retina of 15-month-old $d b /$ $d b$ mice. Considered to be resident macrophages, microglia play an important role in vascular development and remodelling in the central nervous system [42]. In diabetic retinas, dysregulated microglia promote cytokine production, leading 
to chronic inflammation and neurovascular degeneration [43]. In addition, long-term diabetes alters the bone marrow microenvironment, which skews haematopoiesis towards generating more inflammatory monocytes while reducing the number of circulating progenitors [24]. Whether and how increased ER stress and microglial activation in the diabetic retina have an adverse impact on CAC function in vascular repair remains elusive.

Compelling evidence suggests that multiple aetiological factors, including increased oxidative stress, NADPH oxidase activation and an altered nitric oxide pathway, contribute to the defective function of CACs in diabetes $[25,44,45]$. Interestingly, a recent study by Li and associates demonstrated that homocysteine, a risk factor for atherosclerosis, induces the apoptosis of human CACs through an induction of ER stress and CHOP-mediated caspase-3 activation [17]. Our present study represents the first investigation of a role for ER stress in the dysfunction of angiogenic progenitors in diabetes. Our results show that bone marrow-derived EOCs isolated from $d b / d b$ mice or cultured under extended HG conditions exhibit significantly increased ER stress. An interesting finding is the discrepancy in GRP78 levels, which are increased in diabetic EOCs but slightly reduced in HG-treated EOCs. The mechanism underlying this difference is unclear, but it may be attributed to factors related to the complexity of the in vivo situation in contrast to the relatively simplified in vitro condition. Nevertheless, previous work has implicated GRP78 as a key regulator of stem cell survival. For example, the depletion of GRP78 in intestinal stem cells results in a loss of stemness due to increased PERK-eIF2 $\alpha$ UPR [46], while an acute ablation of GRP78 causes a significant reduction in the number of HSCs, and the conditional knockout of GRP78 leads to a constitutive activation of all major UPR pathways and apoptosis in HSCs [47]. Thus, alterations in GRP78 in diabetic and HG-treated EOCs suggest a potential role of this protein in diabetic angiogenic dysfunction, which will be of great interest for future study.

More recently, the pioneering work by van Galen et al has revealed an essential role for the UPR in maintaining the cellular integrity of haematopoietic stem and progenitor cells [48]. A gene expression analysis of lineage-depleted human cord blood cells has shown that the mRNA levels of ATF4, CHOP, PERK and unspliced XBP1 (XBPIu) are upregulated in HSCs but not in downstream progenitors [48]. Furthermore, activation of the PERK-eIF2 $\alpha-$ ATF4-CHOP-GADD3 4 (growth arrest and DNA damage-inducible protein) pathway predisposes HSCs to apoptosis, whereas enhancing GRP78 activity improves repopulation and functioning of the HSCs. In our study, we found that diabetic and HG-treated EOCs produced higher levels of UPR factors in the IRE- $1 \alpha-$ XBP1 and p-eIF $2 \alpha-A T F 4$ pathways. Importantly, the production of CHOP, a major mediator of ER stress-related apoptosis, was significantly increased, as was caspase- 3 activation, suggesting a role of ER stress in diabetes-triggered apoptosis in EOCs. More importantly, our results show that the chemical chaperone PBA inhibits ER stress, which successfully mitigates HG-induced EOC dysfunction and prevents apoptosis. These findings not only elucidate for the first time a crucial role of ER stress in mediating diabetic EOC damage, but also provide a rationale for developing a novel approach to protecting angiogenic progenitors in diabetes by targeting ER stress. Future work will delineate how the UPR pathways are implicated in the diabetes-related dysfunction of CACs and bone marrow progenitors and vascular degeneration.

Acknowledgements The authors thank M. B. Grant (University of Indiana, USA) for critical reading and helpful comments on the manuscript.

Funding This work is supported, in part, by NIH/NEI grants EY019949 and EY025061, ADA research grant \#7-11-BS-182 and an Unrestricted Grant to the Department of Ophthalmology, SUNY-Buffalo, from Research to Prevent Blindness.

Duality of interest The authors declare that there is no duality of interest associated with this manuscript.

Contribution statement MB, JJW and SXZ conceived and designed the experiments. MB, JHM, JS and JJW performed the experiments. All authors analysed and interpreted the data, wrote and revised the manuscript and approved the final version. SXZ is the guarantor of this work.

\section{References}

1. Fong DS, Aiello L, Gardner TW et al (2004) Retinopathy in diabetes. Diabetes Care 27(Suppl 1):S84-87

2. Mohamed Q, Gillies MC, Wong TY (2007) Management of diabetic retinopathy: a systematic review. JAMA J Am Med Assoc 298:902-916

3. Yoder MC (2010) Is endothelium the origin of endothelial progenitor cells? Arterioscler Thromb Vasc Biol 30:1094-1103

4. Asahara T, Murohara T, Sullivan A et al (1997) Isolation of putative progenitor endothelial cells for angiogenesis. Science 275:964-967

5. Tepper OM, Galiano RD, Capla JM et al (2002) Human endothelial progenitor cells from type II diabetics exhibit impaired proliferation, adhesion, and incorporation into vascular structures. Circulation 106:2781-2786

6. Yamaguchi J, Kusano KF, Masuo O et al (2003) Stromal cellderived factor-1 effects on ex vivo expanded endothelial progenitor cell recruitment for ischemic neovascularization. Circulation 107:1322-1328

7. Jarajapu YP, Caballero S, Verma A et al (2011) Blockade of NADP $\mathrm{H}$ oxidase restores vasoreparative function in diabetic $\mathrm{CD} 34+$ cells. Invest Ophthalmol Vis Sci 52:5093-5104

8. Medina RJ, O'Neill CL, O'Doherty TM et al (2013) Ex vivo expansion of human outgrowth endothelial cells leads to IL-8mediated replicative senescence and impaired vasoreparative function. Stem Cells 31:1657-1668

9. McMaster CR (2001) Lipid metabolism and vesicle trafficking: more than just greasing the transport machinery. Biochem Cell Biol Biochim Biol Cell 79:681-692

10. Zhang SX, Sanders E, Wang JJ (2011) Endoplasmic reticulum stress and inflammation: mechanisms and implications in diabetic retinopathy. J Ocul Biol Dis Inform 4:51-61 
11. Zhang SX, Sanders E, Fliesler SJ, Wang JJ (2014) Endoplasmic reticulum stress and the unfolded protein responses in retinal degeneration. Exp Eye Res 125C:30-40

12. Chen Y, Wang JJ, Li J et al (2012) Activating transcription factor 4 mediates hyperglycaemia-induced endothelial inflammation and retinal vascular leakage through activation of STAT3 in a mouse model of type 1 diabetes. Diabetologia 55:2533-2545

13. Zhong Y, Li J, Chen Y, Wang JJ, Ratan R, Zhang SX (2012) Activation of endoplasmic reticulum stress by hyperglycemia is essential for muller cell-derived inflammatory cytokine production in diabetes. Diabetes 61:492-504

14. Li J, Wang JJ, Yu Q, Wang M, Zhang SX (2009) Endoplasmic reticulum stress is implicated in retinal inflammation and diabetic retinopathy. FEBS Lett 583:1521-1527

15. Zhang SX, Ma JH, Bhatta M, Fliesler SJ, Wang JJ (2015) The unfolded protein response in retinal vascular diseases: implications and therapeutic potential beyond protein folding. Prog Retin Eye Res 45C:111-131

16. Zeng L, Zampetaki A, Margariti A et al (2009) Sustained activation of XBP1 splicing leads to endothelial apoptosis and atherosclerosis development in response to disturbed flow. Proc Natl Acad Sci 106:8326-8331

17. Li L, Hu BC, Gong SJ, Yan J (2011) Homocysteine-induced caspase-3 activation by endoplasmic reticulum stress in endothelial progenitor cells from patients with coronary heart disease and healthy donors. Biosci Biotechnol Biochem 75:1300-1305

18. Ii M (2010) Bone marrow-derived endothelial progenitor cells: isolation and characterization for myocardial repair. Methods Mol Biol 660:9-27

19. Krishnamurthy $P$, Thal M, Verma $S$ et al (2011) Interleukin-10 deficiency impairs bone marrow-derived endothelial progenitor cell survival and function in ischemic myocardium. Circ Res 109:1280-1289

20. Zhang Y, Yuen DA, Advani A et al (2012) Early-outgrowth bone marrow cells attenuate renal injury and dysfunction via an antioxidant effect in a mouse model of type 2 diabetes. Diabetes 61:2114-2125

21. Chen C, Cano M, Wang JJ et al (2014) Role of unfolded protein response dysregulation in oxidative injury of retinal pigment epithelial cells. Antioxid Redox Signal 20:2091-2106

22. Yang J, Ii M, Kamei N et al (2011) CD34+ cells represent highly functional endothelial progenitor cells in murine bone marrow. PLoS One 6, e20219

23. Westerweel PE, Teraa M, Rafii S et al (2013) Impaired endothelial progenitor cell mobilization and dysfunctional bone marrow stroma in diabetes mellitus. PLoS One 8, e60357

24. Hazra S, Jarajapu YP, Stepps V et al (2013) Long-term type 1 diabetes influences haematopoietic stem cells by reducing vascular repair potential and increasing inflammatory monocyte generation in a murine model. Diabetologia 56:644-653

25. Marrotte EJ, Chen DD, Hakim JS, Chen AF (2010) Manganese superoxide dismutase expression in endothelial progenitor cells accelerates wound healing in diabetic mice. J Clin Invest 120:4207-4219

26. Takahashi T, Kalka C, Masuda H et al (1999) Ischemia- and cytokine-induced mobilization of bone marrow-derived endothelial progenitor cells for neovascularization. Nat Med 5:434-438

27. Villalvilla A, Moro M, Arruza L, Redondo S, Fernandez-Cruz A, Fernandez-Durango R (2012) Circulating endothelial progenitor cells are reduced in rat oxygen-induced retinopathy despite a retinal SDF-1/CXCR4 and VEGF proangiogenic response. Life Sci 91:264-270

28. Gardner TW, Antonetti DA, Barber AJ, LaNoue KF, Nakamura M (2000) New insights into the pathophysiology of diabetic retinopathy: potential cell-specific therapeutic targets. Diabetes Technol Ther 2:601-608

29. Zeng HY, Green WR, Tso MO (2008) Microglial activation in human diabetic retinopathy. Arch Ophthalmol 126:227-232

30. Gaucher D, Chiappore JA, Paques M et al (2007) Microglial changes occur without neural cell death in diabetic retinopathy. Vis Res 47:612-623

31. Abcouwer SF (2013) Angiogenic factors and cytokines in diabetic retinopathy. J Clin Cellular Immunol. doi:10.4172/2155-9899

32. Marty S, Dusart I, Peschanski M (1991) Glial changes following an excitotoxic lesion in the CNS-I. Microglia/macrophages. Neuroscience 45:529-539

33. Poulaki V, Qin W, Joussen AM et al (2002) Acute intensive insulin therapy exacerbates diabetic blood-retinal barrier breakdown via hypoxia-inducible factor-1alpha and VEGF. J Clin Invest 109:805-815

34. Hoenig MR, Bianchi C, Sellke FW (2008) Hypoxia inducible factor-1 alpha, endothelial progenitor cells, monocytes, cardiovascular risk, wound healing, cobalt and hydralazine: a unifying hypothesis. Curr Drug Targets 9:422-435

35. Chen Y-H, Lin S-J, Lin F-Y et al (2007) High glucose impairs early and late endothelial progenitor cells by modifying nitric oxiderelated but not oxidative stress-mediated mechanisms. Diabetes $56: 1559-1568$

36. Shaw LC, Neu MB, Grant MB (2011) Cell-based therapies for diabetic retinopathy. Curr Diabetes Rep 11:265-274

37. Caballero S, Sengupta N, Afzal A et al (2007) Ischemic vascular damage can be repaired by healthy, but not diabetic, endothelial progenitor cells. Diabetes 56:960-967

38. Yan J, Tie G, Park B, Yan Y, Nowicki PT, Messina LM (2009) Recovery from hind limb ischemia is less effective in type 2 than in type 1 diabetic mice: roles of endothelial nitric oxide synthase and endothelial progenitor cells. J Vasc Surg 50:1412-1422

39. Sivan-Loukianova E, Awad OA, Stepanovic V, Bickenbach J, Schatteman GC (2003) CD34+ blood cells accelerate vascularization and healing of diabetic mouse skin wounds. J Vasc Res 40:368-377

40. Schatteman GC, Hanlon HD, Jiao C, Dodds SG, Christy BA (2000) Blood-derived angioblasts accelerate blood-flow restoration in diabetic mice. J Clin Invest 106:571-578

41. Du M, Wu M, Fu D et al (2013) Effects of modified LDL and HDL on retinal pigment epithelial cells: a role in diabetic retinopathy? Diabetologia 56:2318-2328

42. Arnold T, Betsholtz C (2013) The importance of microglia in the development of the vasculature in the central nervous system. Vasc Cell 5:4

43. Grigsby JG, Cardona SM, Pouw CE et al (2014) The role of microglia in diabetic retinopathy. J Ophthalmol 2014:705783

44. Hamed S, Brenner B, Aharon A, Daoud D, Roguin A (2009) Nitric oxide and superoxide dismutase modulate endothelial progenitor cell function in type 2 diabetes mellitus. Cardiovasc Diabetol 8:56

45. Ebrahimian TG, Heymes C, You D et al (2006) NADPH oxidasederived overproduction of reactive oxygen species impairs postischemic neovascularization in mice with type 1 diabetes. Am J Pathol 169:719-728

46. Heijmans J, van Lidth de Jeude JF, Koo BK et al (2013) ER stress causes rapid loss of intestinal epithelial stemness through activation of the unfolded protein response. Cell Rep 3:1128-1139

47. Wey S, Luo B, Lee AS (2012) Acute inducible ablation of GRP78 reveals its role in hematopoietic stem cell survival, lymphogenesis and regulation of stress signaling. PLoS One 7, e39047

48. van Galen P, Kreso A, Mbong N et al (2014) The unfolded protein response governs integrity of the haematopoietic stem-cell pool during stress. Nature 510:268-272 